\title{
JOURNAL EXCHANGES: Physics journals in East and Central Europe - details and contacts for journal exchanges.
}

The exchange of physics journals is extremely important for east and central European countries as it provides a means to acquire foreign publications. The journals listed below have undertaken to ensure that copies of journals received from outside their respective countries will be made available to the physics community at large (generally by keeping the journals in a centrally located and easily accessible library). Publishers of physics journals are being encouraged to initiate or extend exchange arrangements by contacting the offices listed in the table.

\begin{tabular}{|c|c|c|c|c|c|c|c|c|c|c|}
\hline & Publication & Founded & Publisher & Lang. & $\begin{array}{l}\text { Pages } \\
\text { lissue }\end{array}$ & $\begin{array}{l}\text { Issues } \\
\text { lyear }\end{array}$ & Price & $\begin{array}{l}\text { No. co } \\
\text { printed }\end{array}$ & $\begin{array}{l}\text { pies } \\
\text { exch. }\end{array}$ & Contact for exchanges \\
\hline BU & J Bulgarian J. Phys.* & 1973 & $\begin{array}{l}\text { AS } \\
\text { Sofia Univ. }\end{array}$ & E & 100 & $3-4$ & \$US 60.- & 400 & 300 & $\begin{array}{l}\text { Union of Physicists in } \\
\text { Bulgaria }\end{array}$ \\
\hline $\mathrm{CZ}$ & Czechoslovak J. Phys." & $\begin{array}{l}1872 / \\
1950\end{array}$ & $\begin{array}{l}\text { Inst. of Phys. } \\
\text { AS }\end{array}$ & E & 100 & 12 & SUS 745.- & $400-500$ & 20 & Inst. of Phys. library \\
\hline ES & $\begin{array}{l}\text { Proc. Estonian Acad. of Sci. } \\
\text { Phys., Maths }\end{array}$ & 1951 & $\begin{array}{l}\text { AS } \\
\text { Estonian Phys. Soc. }\end{array}$ & $E, 10 \% R$ & 80 & 4 & \$US 60.- & $?$ & ? & AS library \\
\hline HR & Fizika $A, B$ & 1969 & Croatian Phys. Soc. & $E, 2 \% R$ & 80,80 & 4,4 & SUS 60.- & 650 & 80 & Editorial Office \\
\hline & $\begin{array}{l}\text { Acta Physica Hungarica: } \\
\text { Heavy lon Physics* }\end{array}$ & 1957 & AS & E & 100 & 8 & \$US 192 . & 500 & 50 & Editorial Office \\
\hline LI & Lithuanian J. Phys. ${ }^{\circ}$ & ? & $\begin{array}{l}\text { AS/ } \\
\text { Lithuanian Phys. Soc. }\end{array}$ & $30 \% R$ & $?$ & 6 & $?$ & 200 & 60 & $\begin{array}{l}\text { AS library } \\
\text { Lithuanian Phys. Soc. }\end{array}$ \\
\hline PL & Acta Phys. Polon. A, B* & 1930 & $\begin{array}{l}\text { Inst. of Phys., AS } \\
\text { Polish Phys. Soc. }\end{array}$ & E & 180 & 12 & SUS 354.- & $600-800$ & 65 & $\begin{array}{l}\text { Editorial Office } \\
\text { Inst. of Phys. library }\end{array}$ \\
\hline$R$ & Romanian J. Phys. $^{+}$ & 1955 & AS & $95+\% \mathrm{E}$ & 100 & 10 & Lei $500 .-$ & 250 & $?$ & AS \\
\hline SI & $\begin{array}{l}\text { Obz. za Matematiko in } \\
\text { Fiziko }\end{array}$ & 1951 & SMPAS & SI & 32 & 6 & DM 50.- & 1500 & 60 & SMPAS \\
\hline SL & - Acta Physica Slovaca* & 1950 & Inst. of Physics, AS & $E$ & 60 & 6 & DM 220.- & $?$ & $<10$ & Editorial Office \\
\hline
\end{tabular}

${ }^{\circ}$ English translation available. Languages: E - English; R - Russian. AS: Academy of Science. SMPAS: Society of Mathematicians, Physicists and Astronomers of Slovenia 'EPS Recognized Journal (+: application made; recognition pending).

in borderline areas where it is useful to address a broad audience.

I conclude by greeting my successor, Franco Gianturco, an atomic and molecular physicist from Rome who took over at the beginning of April. Although we come from quite different fields of physics, we have similar ambitions for Europhysics Letters. The various nationalities and scientific specialities of the first four Editors-in-Chief should be a symbol of the openness of our journal. I therefore wish Professor Gianturco every success.

Europhysics Letters and the European Journal of Physics in 1994.

\begin{tabular}{|llc|}
\hline & EPL & EJP \\
\hline Submissions & 772 & 158 \\
Accepted & $59 \%$ & $31 \%$ \\
Europe; N. America & $79 \% ; 11 \%$ & $76 \% ; 8 \%$ \\
Receipt-to-acceptance & 18 weeks & 12 weeks \\
Pages & 2914 & 356 \\
Subjects & pedagogical & all fields* \\
\hline
\end{tabular}

- $56 \%$ condensed matter, $19 \%$ general, $8 \%$ atomic \& molecular, $8 \%$ classical areas.

Editorial Offices (contact for exchanges are underlined)

Bulgarian Journal of Physics*: Physics Faculty, Sofia University, J. Bouchier Blvd. 5, B1126 Sofia. Fax: +359-2-68 9085

Union of Physicists in Bulgaria, Blvd. J. Bourchier 5, BG-1126 Sofia fax: +359-2-68 9085

Czechoslovak Journal of Physics*: Institute of Physics, Academy of Science of the Czech Republic, Na Slovance 2, CZ-180 40 Prague 8. Fax: +42-2-82 1227

Proceedings of the Estonian Academy of Science: Estonian Academy of Science, Estonia pst. 7, EE-0100 Tallinn. Fax.: +372-0142-52 7901

Estonian Academy of Science Library, 10 Rvala Ave., EE-0100 Tallinn

Fizika A, B: POB 162, HR-41000 Zagreb. Fax: +385-1-4321 80

Acta Physica Hungarica: Heavy lon Physics*: Department of Theoretical Physics, Debrecen University, POB 5, H-4010 Debrecen. Fax: +36-52-41 6181

Lithuanian Journal of Physics: Academy of Science of Lithunia, Gedimino pz. 3, LT-2600 Vilnius

Lithunian Physical Society, A. Gostauto 12, LT-2600 Vilnius. Fax: +370-2-61 8464

Acta Physica Polonica*: Institute of Physics, Polish Academy of Sciences, Al. Lotników 32/46, PL-02-668 Warsaw. Fax: +48-22-43 0926

Romanian Journal of Physics ${ }^{+}$: POB MG-6, R-Bucharest/Magurele. Fax: + 40-1-312 2247 Romanian Academy Library, Calea Victoriei 131, R-Bucharest

Obzornik za Matematiko in Fiziko: Society of Mathematicians, Physicists \& Astronomers of Slovenia (SMPAS), POB 64, SI-61111 Ljubjana.Fax: +386-61-21 7281

Acta Physica Slovaca*: Institute of Physics, Slovak Academy of Sciences, Dúbravaská cesta 9, SK-842 28 Bratislava. Fax: +42-7-37 6085

\section{EC WWW SERVERS}

- EC CORDIS database \& Training \& Mobility of Researchers Programme - Research Training Grants information package: $\mathrm{http} / / \mathrm{www}$.cordis.lu/

- I'M Europe (information projects): http://www.echo.lu/

- General information - EUROPA server: http://www.cec.lu/

\section{$28.8 \mathrm{kbps}$ ACCESS}

Many on-line services are starting to offer V.34 $28.8 \mathrm{kbps}$ Internet access: - CompuServe (July '95)

- America Online (April '95)

- Apple Open Transport (April '95)

- EUnet

- Europe Online

- PIPEX (May'95)
- Taligent Common Point (Sept. '95) - Taligent TaIOS (April '96)

\section{TELEPHONING VIA INTERNET}

Modem software now available.

- NetPhone: Electric Magic (San

Francisco, CA, USA)

- Personal Internet Companion Kit:

Camelot Corp. (Dallas, TX, USA)

- VocalTec (Israel)
ONLINE DEGREES/COURSES

- Globewide Network Academy: http://uu-gna.mit.edu:8001/uu-gna/ - Entertaining Science: http://www.lassp.cornell.edu/ - Physics courses: http://www.tc.cornell.edu:80/edu/ MathSciGateway/ - Interactive Physics Problems Set: http://128.32.163.55/Vol1/ 